rehospitalization in the following year in the non-screened group $(p<0.001)$, but not in the screened group ( $p=0.750$ and $p=0.066$ respectively).

Conclusion: Our screening and prevention program was associated with a reduction in hospitalizations in the following year and a decrease in the risk of re-hospitalization compared to unscreened patients with IRD. This suggests a positive impact of performing systematic screening for multi-morbidities in IRD patients.

Acknowledgements: We thank Pfizer for their financial support

Disclosure of Interests: guillaume decarriere: None declared, Jenica PASTOR: None declared, David DEMOULIN: None declared, Gael Mouterde Speakers bureau: Bristol-Myers Squibb; Gilead; Janssen; Lilly; Merck; Novartis; Pfizer; RocheChugai; and Sanofi, Grant/research support from: Pfizer, Cédric Lukas Speakers bureau: Abbvie, Amgen, Janssen, Lilly, MSD, Novartis, Pfizer, Roche-Chugai, UCB, Consultant of: Abbvie, Amgen, Janssen, Lilly, MSD, Novartis, Pfizer, Roche-Chugai, UCB, Grant/research support from: Pfizer, Novartis and Roche-Chugai, Bernard Combe Speakers bureau: AbbVie; Bristol-Myers Squibb; Gilead; Janssen; Lilly; Merck; Novartis; Pfizer; Roche-Chugai; and Sanofi, Consultant of: AbbVie; Bristol-Myers Squibb; Gilead; Janssen; Lilly; Merck; Novartis; Pfizer; Roche-Chugai; and Sanofi, Grant/research support from: Novartis, Pfizer, and Roche-Chugai, Grégoire Mercier: None declared, Jacques Morel Speakers bureau: AbbVie; Bristol-Myers Squibb; Gilead; Janssen; Lilly; Merck; Novartis; Pfizer; Roche-Chugai; and Sanofi, Consultant of: AbbVie; Bristol-Myers Squibb; Gilead; Janssen; Lilly; Merck; Novartis; Pfizer; Roche-Chugai; and Sanofi, Grant/research support from: Novartis, Pfizer, and Roche-Chugai, Claire Daien Speakers bureau: Pfizer, Roche-Chugai, Fresenius, BMS, MSD, Lilly, Novartis, Galapagos, Consultant of: Abivax, Abbbvie, BMS, Roche-Chugai, Grant/research support from: Pfizer, roche-chugai, fresenius, MSD DOI: 10.1136/annrheumdis-2021-eular.3454

\section{OP0215 MENTAL HEALTH, SLEEP AND COGNITION CHARACTERISTICS IN RHEUMATOID ARTHRITIS AND ASSOCIATIONS WITH RHEUMATOID FACTOR STATUS IN THE UK BIOBANK}

I. Stanciu ${ }^{1}$, S. Siebert ${ }^{2}$, D. Mackay ${ }^{1}$, D. Lyall ${ }^{1} .{ }^{1}$ University of Glasgow, Public Health, Glasgow, United Kingdom; ${ }^{2}$ University of Glasgow, Infection Immunity \& Inflammation, Glasgow, United Kingdom

Background: There are significant gaps in the literature regarding sleep, mental health, and cognition for people with rheumatoid arthritis (RA) despite being important aspects of patients' overall quality of life. Similarly, there is a lack of understanding about the role of rheumatoid factor (RF) on these domains.

Objectives: The aim of the current study was to characterize mental health, cognition, and sleep variables in people with RA and to compare these associations in people with positive $R F(R F+)$ and negative $R F(R F-)$ in a large population cohort. Methods: This cross-sectional study used baseline data from the UK Biobank cohort $(n=502,506)$ to compare people with and without $R A$ and people that are $\mathrm{RF}+$ versus RF- on a variety of sociodemographic, lifestyle, illness-related factors and depression, neuroticism, performance on cognitive tests and sleep-related factors. Logistic regression analyses were also performed to determine whether RF seropositivity was associated with mental health, cognition, and sleep variables. We adjusted for the covariates of age, sex, ethnicity, deprivation index, smoking status, BMI and alcohol intake.

Results: In this sample 5,907 people self-reported having RA (1.17\%), of which $74 \%$ were RF- and $26 \%$ were RF+. There were significant differences $(p<0.05)$ between people with and without RA for depression, neuroticism, nap during the day, getting up in the morning, insomnia, reaction time, fluid intelligence and prospective memory. There were significant differences $(p<0.05)$ between RF+ and RF- people for depression, neuroticism, sleep duration, nap during the day, getting up in the morning, insomnia, and reaction time. In the unadjusted regression analyses neuroticism $(B=-0.06, S E=0.01, p<0.001)$, sleep duration $(B=0.02, S E=0.005, p<0.001)$, nap during the day $(\mathrm{OR}=1.28,95 \% \mathrm{Cl}: 1.02-1.65, \mathrm{p}<0.05)$ and reaction time $(B=4.55$, $S E=0.53, p<0.001)$ were significantly associated with RF status. After adjusting for covariates, only sleep duration $(B=0.01, S E=0.005, p<0.01)$ remained significant. Conclusion: The current study suggests that RA diagnosis and RF status are associated with differences in mental health, sleep, and cognition, highlighting the importance of addressing these aspects in clinical settings and future research.

Disclosure of Interests: None declared

DOI: 10.1136/annrheumdis-2021-eular.1767

OP0216

TRENDS IN OCCURRENCE OF DEMENTIA IN PATIENTS WITH RHEUMATOID ARTHRITIS: A POPULATION-BASED COHORT STUDY, 1980-2009

V. Kronzer ${ }^{1}$, C. S. Crowson ${ }^{1}$, J. M. Davis III ${ }^{1}$, M. Vassilaki ${ }^{2}$, M. Mielke ${ }^{2}$, E. Myasoedova'. ${ }^{1}$ Mayo Clinic, Rheumatology, Rochester, United States of America; ${ }^{2}$ Mayo Clinic, Health Sciences Research, Rochester, United States of America

Background: Some cross-sectional studies show increased odds of cognitive impairment and dementia in patients with rheumatoid arthritis (RA) compared to the general population, while others show the reverse. Furthermore, existing studies have not evaluated trends in incidence of dementia on a longitudinal basis. Objectives: We aimed to assess the incidence of dementia over time in patients with incident RA and to compare it to those of population-based comparators. Methods: This population-based, retrospective cohort study included Olmsted County residents with incident RA and non-RA individuals matched on age, sex, and calendar year. All RA cases met 1987 ACR criteria for RA between 1980 and 2009. Index date was the date of RA criteria fulfillment or a corresponding date for referents. All individuals were followed until death, migration, or 12/31/2019. For sensitivity analyses, follow-up of each decade was truncated at eleven years to make the length of follow-up comparable (e.g., the 1980-89 cohort was truncated at 12/31/1999). Incident dementia was defined as an ICD-9/10 code for dementia Patients with dementia prior to RA incidence/index date were excluded. Cox proportional hazards models calculated hazard ratios $(\mathrm{HR})$ with $95 \%$ confidence intervals (Cl) for incident dementia by decade, adjusting for age and sex. The cumulative incidence of dementia was assessed, adjusting for the competing risk of death. Results: The study included 895 persons with RA (mean age 55.3 years; $69 \%$ female) followed up for a median of 15.2 years. The 10-year cumulative incidence of dementia in these individuals during the 1980 s was $12.7 \%(95 \% \mathrm{Cl} 7.9-15.7 \%)$ 1990s was $7.2 \%$ (95\% Cl 3.7-9.4\%), and 2000 s was $6.2 \%(95 \% \mathrm{Cl} 3.6-7.8 \%)$ Patients with incident RA in 2000-09 had markedly lower cumulative incidence of dementia than patients diagnosed in the 1980 s ( $\mathrm{HR} 0.57 ; 95 \% \mathrm{Cl} 0.33-0.98)$. Patients with incident RA were then compared to population-based comparators without RA ( $\mathrm{N}=880$, mean age 55.2 years; $68 \%$ female) followed up for a median of 16.4 years. The 10-year cumulative incidence of dementia in these individuals in the 1980s was $9.3 \%(95 \% \mathrm{Cl} 4.6-11.9 \%)$, in the 1990 s was $5.0 \%(95 \% \mathrm{Cl} 2.2-6.3 \%)$ and in the 2000 s was $7.1 \%(95 \% \mathrm{Cl} 4.3-8.9 \%)$. Overall, the risk of dementia in RA patients was significantly higher than in the non-RA persons (HR 1.38; 95\% $\mathrm{Cl} 1.04-1.83$ ). When subdivided by decade, the risk of dementia in patients diagnosed with RA was higher than non-RA comparators in the 1980s and 1990s but not 2000s (Figure 1). Sensitivity analysis with truncated follow-up yielded similar results.
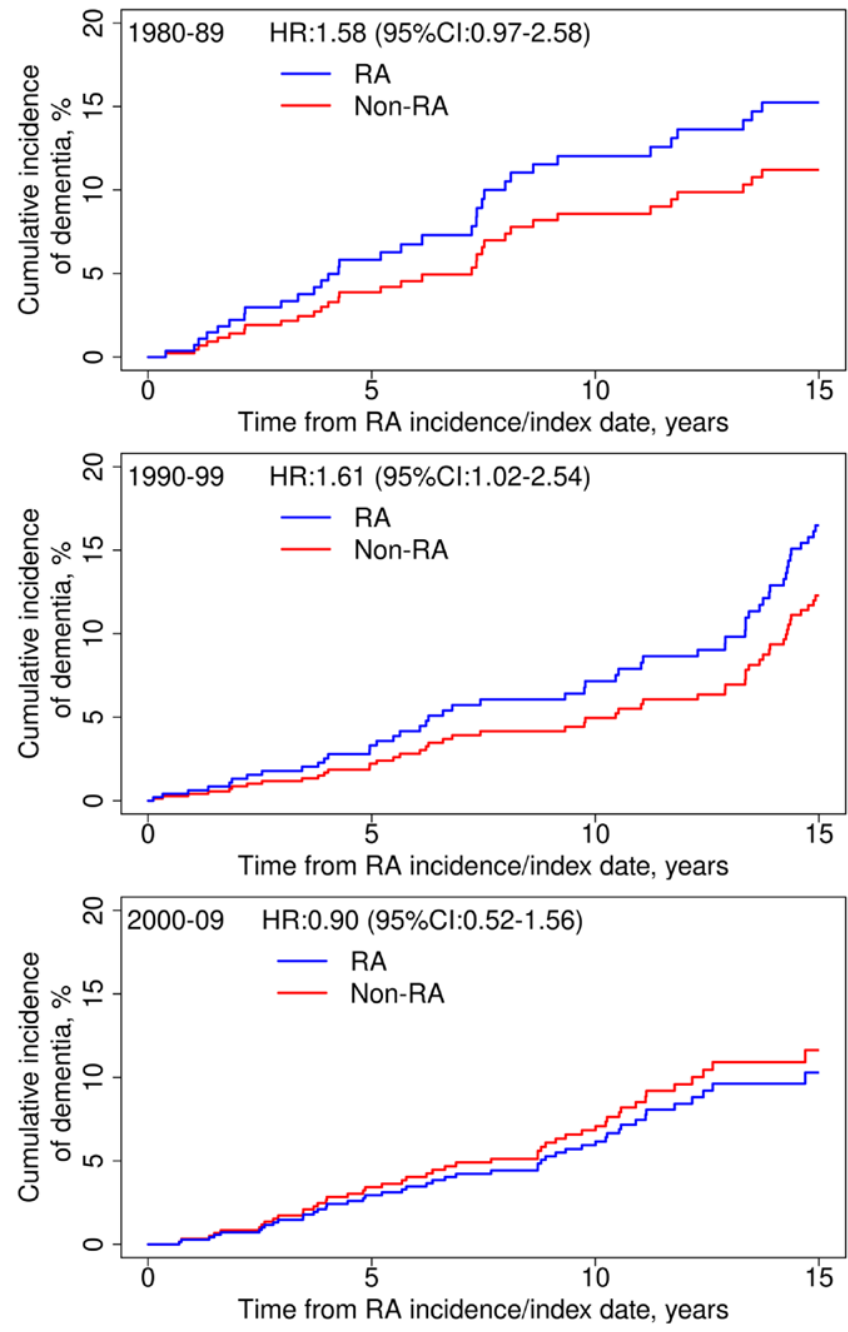

Figure 1. Cumulative incidence of dementia (based on ICD 9/10 codes) versus age-and sexmatched non-RA comparators by decade of RA incidence/index. 
Conclusion: Our findings show substantial decline in risk of dementia in patients with RA onset in the 2000 s as compared to 1980 s, including when compared to the general population comparators. This decline coincides with the advent of novel biologic treatments for RA. Further studies should investigate this association using manual verification rather than billing codes for dementia, and should also elucidate the role of inflammation, autoimmunity, and anti-rheumatic treatments in risk of dementia.

Acknowledgements: This work was funded by grants from the National Institutes of Health, NIAMS (R01 AR46849) and NIA (R01 AG068192, R01 AG034676). Disclosure of Interests: Vanessa Kronzer: None declared, Cynthia S. Crowson: None declared, John M Davis III Grant/research support from: Pfizer, Maria Vassilaki Shareholder of: Abbott Laboratories, Johnson and Johnson, Medronic and Amgen, Grant/research support from: Roche, Biogen, Michelle Mielke Consultant of: Biogen and Brain Protection Company, Elena Myasoedova: None declared

DOI: 10.1136/annrheumdis-2021-eular.150

\section{OP0217 IS METACARPOPHALANGEAL-JOINT PAIN AS AN EARLY SYMPTOM OF PATIENTS AT RISK FOR PROGRESSION TO INFLAMMATORY ARTHRITIS EXPLAINED BY MRI-DETECTED SUBCLINICAL INFLAMMATION? - A LARGE OBSERVATIONAL STUDY}

Q. Dumoulin ${ }^{1}$, X. Matthijssen ${ }^{1}$, F. Wouters ${ }^{1}$, A. Van der Helm - van $\mathrm{Mil}^{1,2}$, E. Niemantsverdriet ${ }^{1} .{ }^{1}$ Leiden University Medical Center (LUMC), Rheumatology, Leiden, Netherlands; ${ }^{2}$ Erasmus MC, Rheumatology, Rotterdam, Netherlands

Background: Pain in metacarpophalangeal (MCP)-joints in patients presenting with clinically suspect arthralgia (CSA) is one of the clinical features by which patients are considered at risk for progression to inflammatory arthritis (IA). As such this symptom is characteristic for CSA and therefore part of a list of clinical parameters determined by an EULAR-taskforce to identify a more homogeneous group of patients within CSA (the EULAR definition of arthralgia suspicious for progression to RA). MRI-detected subclinical inflammation is known to be present in patients with CSA. In general, arthralgia in CSA can be explained by this subclinical inflammation, however to date, the association of subclinical inflammation with pain in MCP-joints specifically is not clear. Subsequently, it is unknown whether this association differs pertinently when investigated with self-reported pain, or with pain in the form of tenderness at physical examination. Objectives: This study will investigate whether MCP-pain and MCP-joint tenderness are associated with MRI-detected subclinical inflammation in patients with CSA, and more specifically those who have progressed to IA

Methods: Between April 2012- February 2019, 602 patients were consecutively included in the Leiden clinically suspect arthralgia (CSA)-cohort. Follow-up ended when patients developed clinically apparent IA (determined at physical examination), or else after 2-years (median follow-up time 25 months). MCP-joints were assessed for self-reported joint pain by the patient using a mannequin and subsequently for joint tenderness by physical examination. Baseline unilateral MRIs of the MCP (2-5)-joints were scored by two readers, blinded for clinical data, on subclinical inflammation (synovitis, tenosynovitis, osteitis). Associations between MCP-pain or MCP-joint tenderness and MRI-detected subclinical inflammation were studied at patient level by logistic regression analyses, entering the mentioned MRI-detected features separately (univariable) and together (multivariable). Results: $33 \%$ of 227 patients with self-reported MCP-pain had MRI-detected subclinical inflammation and $38 \%$ of 226 patients with MCP-joint tenderness had MRI-detected subclinical inflammation. Self-reported MCP-joint pain was univariable associated with subclinical inflammation and synovitis in particular (OR 2.00, 95\% Cl: 1.21-3.30, OR 2.87, 95\% Cl: 1.29-6.39). In multivariable analysis this MCP-pain was associated with synovitis (OR 2.54, 95\% Cl: 1.12-5.77). MCP-joint tenderness was univariable associated with subclinical inflammation, and synovitis and tenosynovitis in particular (OR 1.84, 95\% Cl: $1.29-2.63$, OR $1.76,95 \% \mathrm{Cl}$ : $1.10-2.81$, OR $1.69,95 \% \mathrm{Cl}: 1.12-2.55$, respectively). In multivariable analysis, tenosynovitis remained significant (OR 1.54, 95\% Cl: 1.00-2.36). Of all patients with self-reported MCP-joint pain who developed IA, 50\% had MRI-detected subclinical inflammation. For MCP-joint tenderness this was $61 \%$. Patients with MCP-joint tenderness without subclinical inflammation who developed IA, developed clinical arthritis at a joint that was not scanned (85\%), hence they may have had subclinical inflammation that was not imaged. The other $15 \%$ did develop arthritis in an MCP-joint, suggesting that subclinical inflammation developed after CSA-onset. Conclusion: Arthralgia in the MCP-joints is associated with subclinical inflammation in CSA, in particular with synovitis and tenosynovitis. The prevalence of subclinical inflammation is highest for tender joints at physical examination; this can be acknowledged when applying the EULAR definition of arthralgia suspicious for progression to RA.

Disclosure of Interests: None declared

DOI: 10.1136/annrheumdis-2021-eular.1597

\section{Unrevealing the impact of PsA and comorbidity prevention}

\section{OP0218 \\ MORTALITY IN PATIENTS WITH PSORIATIC ARTHRITIS} IN SWEDEN

S. Exarchou ${ }^{1}$, D. DI Giuseppe ${ }^{2}$, G. M. Alenius ${ }^{3}$, E. Klingberg ${ }^{4,5}$, V. Sigurdardottir ${ }^{6,7}$ S. Wedrén ${ }^{2,8}$, U. Lindström ${ }^{5}$, C. Turesson ${ }^{1}$, L. T. H. Jacobsson ${ }^{5}$, J. Askling ${ }^{2}$, J. K. Wallman ${ }^{9,10}$. 'Lund University, Department of Clinical Sciences Malmö, Rheumatology, Lund, Sweden; ${ }^{2}$ Karolinska Institutet, Department of Medicine Solna, Clinical Epidemiology Division, Stockholm, Sweden; ${ }^{3}$ Umeå University, Department of Public Health and Clinical Medicine/Rheumatology, Umeå, Sweden; ${ }^{4}$ Sahlgrenska University Hospital, Department of Rheumatology, Gothenburg, Sweden; ${ }^{5}$ Sahlgrenska Academy, University of Gothenburg, Department of Rheumatology and Inflammation Research, Gothenburg, Sweden; ${ }^{6}$ Falun Hospital, Department of Rheumatology, Falun, Sweden; ${ }^{7}$ Uppsala University, Centre for Clinical Research Dalarna, Uppsala, Sweden; ${ }^{8}$ Karolinska University Hospital, Department of Rheumatology, Stockholm, Sweden; ${ }^{9}$ Skåne University Hospital, Department of Rheumatology, Lund, Sweden; ${ }^{10}$ Lund University, Department of Clinical Sciences Lund, Rheumatology, Lund, Sweden

Background: In contrast to the increased mortality reported in other inflammatory diseases such as rheumatoid arthritis and psoriasis, prior mortality studies in psoriatic arthritis (PsA) have shown inconsistent results.

Objectives: To compare all-cause mortality between PsA patients in Sweden and matched general population controls, and to describe cause of death distributions in the two groups.

Methods: All individuals in Sweden with $\geq 1$ main diagnosis of PsA (ICD-10: L40.5 M07.0-M07.3) from outpatient visits to rheumatology or internal medicine clinics a age $\geq 18$ years $(y)$ 2001-2017 were identified from the Swedish National Patient Register. Each case was matched to 5 general population controls based on sex, county and age in the year of the first registered arthritis diagnosis for the case. Cases and controls were followed from 1 Jan, 2007, or from first PsA diagnosis thereafter for index cases, until first occurrence of death (data from the Swedish Cause of Death Register), emigration or $31 \mathrm{Dec}, 2018$. Mortality was assessed overall, as well as stratified by sex (45\% males) and disease duration (PsA diagnosis prior to 2007 [38\% of cases] vs. 2007-2017), using matched Cox proportional hazard regression, or - in case the Cox assumption regarding proportionality did not hold - matched Breslow test. To account for potential PsA misclassification (in a previous validation study, $86 \%$ of 400 cases fulfilled PsA classification criteria), a sensitivity analysis was performed by randomly replacing $20 \%$ of cases with one of their own controls. Moreover, incidence rate ratios (IRR) of death were calculated overall and stratified by sex, disease duration and age. Finally, causes of death (from the Cause of Death Register) were described for PsA cases and controls.

Results: Over the 12y follow-up, 3121 deaths occurred among 33036 PsA cases (268 402 person-years at risk) and 12884 deaths among 161144 controls (1 302 250 person-years), resulting in an increased mortality among the PsA cases (HR 1.11 [95\% Cl 1.07-1.16], $\mathrm{p}<0.001$, Figure and Table; sensitivity analysis HR 1.09 [1.05-1.14]). The increased mortality was seen mainly among female PsA cases and among cases with longer disease duration (Figure; Table). IRR:s of death were significantly increased for all ages except $<40 \mathrm{y}$, with the numerically highest point-estimates for ages 40-49y and 50-59y (Table). Cause of death frequencies among the PsA cases/controls: cardiovascular disease $29 / 27 \%$; diabetes mellitus $2.1 / 2.5 \%$; chronic kidney disease $0.4 / 0.3 \%$; infection $5.7 / 4.5 \%$; chronic pulmonary disease $5.1 / 4.1 \%$; malignancy $29 / 34 \%$; suicide $2.3 / 2.0 \%$; other $27 / 26 \%$.

Conclusion: In this nationwide $12 \mathrm{y}$ assessment, the mortality risk among PsA patients in Sweden was increased by around $10 \%$ as compared to the general population, mainly driven by increased risks among females and patients with longer disease duration. Cause of death distributions were numerically similar between PsA cases and controls.

References:
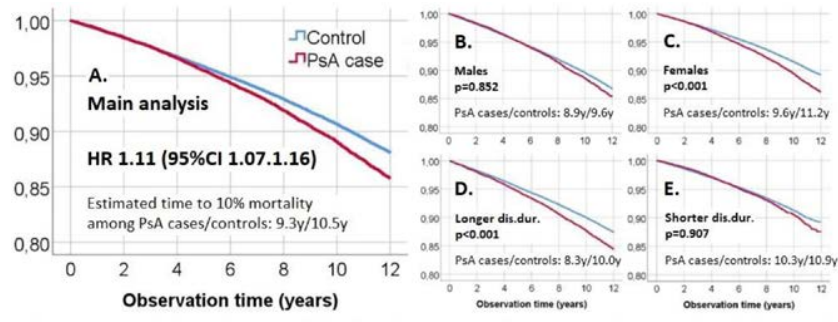

Figure. Kaplan-Meier survival curves displaying all-cause mortality among PsA cases and matched general population controls in Sweden overall (A) and in subgroups (B-E). Estimated time to $10 \%$ mortality are displayed in the respective graphs. A. Main analysis. B. Males, analysed by Breslow test. C. Females, HR disp (95\% 1 16-130) D. duration, analysed by Breslow test. Dis.dur, disease duration; PSA, psoriatic arthritis; y, years. 